\title{
On the Impact of System Resource Constraints on Wireless Relaying Channels
}

\author{
John Boyer, David D. Falconer, Halim Yanikomeroglu \\ Broadband Communications and Wireless Systems Centre \\ Department of Systems and Computer Engineering \\ Carleton University, Ottawa, Canada \\ $\{$ jboyer, ddf, halim\}@sce.carleton.ca
}

\begin{abstract}
This paper considers the impact of various system resource constraints on the connectivity and performance of relaying channels. This consideration is motivated by recent findings indicating that the performance of wireless relaying networks can be increased through the application of distributed spatial diversity techniques (ex. multi-user diversity $[1,5]$, multihop diversity $[2,3]$, cooperative diversity [7-12]) that rely on the mesh connectivity between wireless terminals. Relevant system resource constraints are introduced, constraint combinations are analyzed, and resultant system connectivity models are derived and compared via simulation results. The constraints considered are the available number of orthogonal relaying channels, the ability of relays to diversity combine signals, the ability of the destination to diversity combine signals, the ability of receivers to diversity combine signals on multiple orthogonal channels, and the ability of transmitters to transmit signals on multiple orthogonal channels. The analysis of these system resource constraints is applicable to both amplified (non-regenerative, amplify-and-forward) relaying and decoded (regenerative, decode-and-forward) relaying.
\end{abstract}

Index Terms - multihop relaying, distributed spatial diversity, multihop diversity, cooperative diversity, mesh networks

\section{INTRODUCTION}

Recent findings in the literature have shown that the performance of wireless relaying networks can be increased through the application of distributed spatial diversity techniques that rely on the mesh connectivity between wireless mobile terminals [1-5,8-11,13-14]. Multi-user diversity [1,5] achieves spatial diversity by relaying the signal along multiple routes in parallel. Multihop diversity $[2,3]$ achieves spatial diversity from the concurrent reception of signals that have been transmitted by multiple relays in serial along a single primary route. Cooperative diversity [811,13-14] achieves spatial diversity by sharing information between the source terminal and cooperating relay terminals such that each user of the cooperation group sends information to the destination using all of the cooperating terminals. The symbol error probability of parallel combinations of serial relaying channels is derived in [12]. The aggregate SNR of arbitrarily connected amplified relaying channels is analyzed in [4].

Each of these distributed spatial diversity techniques places different requirements on the available system resources due to a reliance on mesh connectivity between terminals. Therefore, system resource constraints that limit the terminal connectivity constrain the distributed spatial diversity techniques that can be applied. The system resource constraints considered in this paper are the available number

This work was supported in part by the Natural Sciences \& Engineering Research Council of Canada (NSERC) under participation in project WINNER (Wireless World Initiative New Radio) - www.ist-winner.org. of orthogonal relaying channels, the ability of relays to diversity combine incident signals, the ability of the destination to diversity combine incident signals, the ability of receivers to combine signals on multiple orthogonal channels, and the ability of transmitters to transmit signals on multiple orthogonal channels.

Analysis of the impact of these system resource constraints on the connectivity and performance of relaying channels proceeds according to the following methodology. The relevant system resource constraints are introduced and motivated, all possible constraint combinations are analyzed and reduced where appropriate, the set of system connectivity models resulting from these constraint combinations are derived and associated with their minimum cost constraint sets, and these system connectivity models are compared via simulation results.

\section{System ResourCE CONSTRAINTS}

The system resource constraints are described in detail in this section. The motivation for each constraint is discussed in terms of system complexity and cost. Options for each constraint are introduced, along with their corresponding relative cost and connectivity impact. In all cases, constraint options with lower cost have higher connectivity impact. Connectivity impact is defined in comparison to a fully connected system with signal links between all terminals.

Number Channels Available (NCA): This constraint defines the number of orthogonal relaying channels available for the transmission of a signal between a single sourcedestination pair. The half-duplex nature of wireless terminal hardware requires that each relay transmit and receive with different channels, implying a minimum of two orthogonal channels. Use of more than two orthogonal channels increases the system cost since more bandwidth is necessary for each source-destination pair.

- N Channels Available (NCA): The source and each relay transmit using individual separate orthogonal channels. There is no connectivity impact.

- 2 Channels Available (2CA): The source and all relays transmit using the same two orthogonal channels. The connectivity impact is that receivers may only be connected to transmitters on the opposite channel (an odd number of hops away).

Relay Combination (RC): This constraint defines the ability of relay terminals to diversity combine incident signals from multiple preceding terminals. Use of relay diversity combination increases the system cost since separate combination hardware is required for each relayed signal.

- Relay Combination (RC): Relays are able to diversity combine incident signals from multiple preceding terminals. There is no connectivity impact. 
- No Relay Combination (NRC): Relays are not able to diversity combine incident signals from multiple preceding terminals. The connectivity impact is that relays may only be connected to one transmitter.

Destination Combination (DC): This constraint defines the ability of destination terminals to diversity combine incident signals from multiple preceding terminals. Use of destination diversity combination increases the system cost since combination hardware is required for received signals.

- Destination Combination (DC): Destinations are able to diversity combine incident signals from multiple preceding terminals. There is no connectivity impact.

- No Destination Combination (NDC): Destinations are not able to diversity combine incident signals from multiple preceding terminals. The connectivity impact is that destinations may only be connected to one transmitter.

Multiple Channel Reception (MCR): This constraint defines the ability of receivers to combine signals that are on multiple different orthogonal channels. Use of multiple channel reception increases the system cost since more complex multiple channel combination hardware is required.

- Multiple Channel Reception (MCR): Receivers are able to diversity combine signals on different orthogonal channels. There is no connectivity impact.

- Single Channel Reception (SCR): Receivers are not able to diversity combine signals on different orthogonal channels. The connectivity impact is that receivers may only be connected to a subset of transmitters that use one common channel.

Multiple Channel Transmission (MCT): This constraint defines the ability of transmitters to concurrently transmit on multiple different orthogonal channels. Use of multiple channel transmission increases the system cost since more complex multiple channel transmission hardware is required.

- Multiple Channel Transmission (MCT): Transmitters are able to concurrently transmit on multiple orthogonal channels. There is no connectivity impact.

- Single Channel Transmission (SCT): Transmitters are not able to concurrently transmit on multiple orthogonal channels. The connectivity impact is that transmitters may only be connected to a subset of receivers that use one common channel.

\section{Constraint COMBinations}

The possible system resource constraint combinations are analyzed in this section. The combinations are reduced where practical considerations deem appropriate. A set of resultant system connectivity models is derived from the combinations. A detailed derivation of the connectivity models is not included due to space limitations. Fig. 1 summarizes the system resource constraint combinations along with the corresponding resultant system connectivity models. The acronyms used in Fig. 1 are those introduced in the previous section. The following terminology is applied when describing the resultant system connectivity models:

- Single Relay (1R): Relays connected to one transmitter.

- 2Chnl Relay (2R): Relays connected to the subset of transmitters on one channel.
- Full Relay (FR): Relays connected to all transmitters.

- Single Destination (1D): Destination connected to one transmitter.

- 2Chnl Destination (2D): Destination connected to the subset of transmitters on one channel.

- Full Destination (FD): Destination connected to all transmitters.

- 2Chnl Source (2S): Source connected to a subset of receivers on both channels.

- Full Source (FS): Source connected to all receivers.

\begin{tabular}{|c|c|c|c|c|c|}
\hline NCA & RC & DC & MCR & MCT & Resultant Model \\
\hline NCA & $\mathrm{RC}$ & $\mathrm{DC}$ & MCR & MCT & FRFD \\
\hline $\mathrm{NCA}$ & $\mathrm{RC}$ & DC & MCR & SCT & FRFD \\
\hline $\mathrm{NCA}$ & $\mathrm{RC}$ & $\mathrm{DC}$ & SCR & MCT & FRFD \\
\hline $\mathrm{NCA}$ & $\mathrm{RC}$ & $\mathrm{DC}$ & SCR & SCT & 1R1D $^{1}$ \\
\hline $\mathrm{NCA}$ & $\mathrm{RC}$ & NDC & MCR & MCT & FR1D $^{2}$ \\
\hline $\mathrm{NCA}$ & $\mathrm{RC}$ & $\mathrm{NDC}$ & MCR & SCT & FR1D $^{2}$ \\
\hline $\mathrm{NCA}$ & $\mathrm{RC}$ & $\mathrm{NDC}$ & SCR & MCT & FR1D $^{2}$ \\
\hline $\mathrm{NCA}$ & $\mathrm{RC}$ & NDC & SCR & SCT & $1 \mathrm{R}^{1} \mathrm{D}^{1,2}$ \\
\hline $\mathrm{NCA}$ & $\mathrm{NRC}$ & $\mathrm{DC}$ & MCR & MCT & 1RFD \\
\hline $\mathrm{NCA}$ & $\mathrm{NRC}$ & $\mathrm{DC}$ & MCR & SCT & 1RFD \\
\hline $\mathrm{NCA}$ & $\mathrm{NRC}$ & $\mathrm{DC}$ & SCR & $\mathrm{MCT}$ & 1RFD \\
\hline $\mathrm{NCA}$ & $\mathrm{NRC}$ & $\mathrm{DC}$ & SCR & SCT & 1R1D $^{1}$ \\
\hline $\mathrm{NCA}$ & $\mathrm{NRC}$ & NDC & MCR & MCT & 1R1D \\
\hline NCA & $\mathrm{NRC}$ & NDC & MCR & SCT & 1R1D \\
\hline $\mathrm{NCA}$ & $\mathrm{NRC}$ & $\mathrm{NDC}$ & SCR & MCT & 1R1D \\
\hline $\mathrm{NCA}$ & $\mathrm{NRC}$ & $\mathrm{NDC}$ & SCR & SCT & 1R1D \\
\hline $2 \mathrm{CA}$ & $\mathrm{RC}$ & $\mathrm{DC}$ & MCR & MCT & 2RFDFS \\
\hline $2 \mathrm{CA}$ & $\mathrm{RC}$ & $\mathrm{DC}$ & MCR & SCT & 2RFD \\
\hline $2 \mathrm{CA}$ & $\mathrm{RC}$ & $\mathrm{DC}$ & SCR & MCT & 2R2DFS \\
\hline $2 \mathrm{CA}$ & $\mathrm{RC}$ & $\mathrm{DC}$ & SCR & SCT & 2R2D \\
\hline $2 \mathrm{CA}$ & $\mathrm{RC}$ & $\mathrm{NDC}$ & MCR & MCT & 2R1D2S ${ }^{2}$ \\
\hline $2 \mathrm{CA}$ & $\mathrm{RC}$ & $\mathrm{NDC}$ & MCR & SCT & 2R1D ${ }^{2}$ \\
\hline $2 \mathrm{CA}$ & $\mathrm{RC}$ & NDC & SCR & MCT & 2R1D2S ${ }^{2}$ \\
\hline $2 \mathrm{CA}$ & $\mathrm{RC}$ & NDC & SCR & SCT & $2 \mathrm{R} \mathrm{D}^{2}$ \\
\hline $2 \mathrm{CA}$ & $\mathrm{NRC}$ & $\mathrm{DC}$ & MCR & MCT & 1RFD \\
\hline $2 \mathrm{CA}$ & $\mathrm{NRC}$ & $\mathrm{DC}$ & MCR & SCT & 1RFD \\
\hline $2 \mathrm{CA}$ & $\mathrm{NRC}$ & $\mathrm{DC}$ & SCR & MCT & 1R2D2S \\
\hline $2 \mathrm{CA}$ & $\mathrm{NRC}$ & $\mathrm{DC}$ & SCR & SCT & 1R2D \\
\hline $2 \mathrm{CA}$ & $\mathrm{NRC}$ & $\mathrm{NDC}$ & MCR & MCT & 1R1D \\
\hline $2 \mathrm{CA}$ & $\mathrm{NRC}$ & NDC & MCR & SCT & 1R1D \\
\hline $2 \mathrm{CA}$ & $\mathrm{NRC}$ & $\mathrm{NDC}$ & SCR & MCT & 1R1D \\
\hline $2 \mathrm{CA}$ & $\mathrm{NRC}$ & $\mathrm{NDC}$ & SCR & SCT & 1R1D \\
\hline
\end{tabular}

Fig. 1. System Resource Constraint Combinations

1 Constraint combinations with destination and/or relay diversity combination but single channel reception and single channel transmission actually achieve less connectivity when each relay transmits on a separate orthogonal channel than when the source and all relays transmit on the same two channels. Intelligent use of the available channels results in identical connectivity to a system constrained to two available channels.

2 Although not practical in mobile relaying networks, constraint combinations with relay diversity combination but not destination diversity combination are of interest for fixed relaying networks where it is expected that fixed relays will have less resource constraints than mobile destinations.

\section{RESUltant System CONNECTIVITY MODELS}

The set of system connectivity models resulting from the system resource constraint combinations are described in this section. The set of constraints that achieves the corresponding system connectivity models while minimizing the system cost (the minimum cost constraint set) is presented, along with a series of graphical examples that clearly illustrate the differences in system connectivity between the models.

Fig. 2 summarizes the minimum cost constraint sets for the system connectivity models. 


\begin{tabular}{|l|l|}
\hline \multicolumn{1}{|c|}{ System Connectivity Model } & \multicolumn{1}{c|}{ Minimum Cost Constraint Set } \\
\hline 1R1D & $\{2 \mathrm{CA}, \mathrm{NRC}, \mathrm{NDC}, \mathrm{SCR}, \mathrm{SCT}\}$ \\
\hline 1R2D & $\{2 \mathrm{CA}, \mathrm{NRC}, \mathrm{DC}, \mathrm{SCR}, \mathrm{SCT}\}$ \\
\hline 1R2D2S & $\{2 \mathrm{CA}, \mathrm{NRC}, \mathrm{DC}, \mathrm{SCR}, \mathrm{MCT}\}$ \\
\hline 1RFD & $\{2 \mathrm{CA}, \mathrm{NRC}, \mathrm{DC}, \mathrm{MCR}, \mathrm{SCT}\}$ \\
\hline 2R1D & $\{2 \mathrm{CA}, \mathrm{RC}, \mathrm{NDC}, \mathrm{SCR}, \mathrm{SCT}\}$ \\
\hline 2R1D2S & $\{2 \mathrm{CA}, \mathrm{RC}, \mathrm{NDC}, \mathrm{SCR}, \mathrm{MCT}\}$ \\
\hline 2R2D & $\{2 \mathrm{CA}, \mathrm{RC}, \mathrm{DC}, \mathrm{SCR}, \mathrm{SCT}\}$ \\
\hline 2R2DFS & $\{2 \mathrm{CA}, \mathrm{RC}, \mathrm{DC}, \mathrm{SCR}, \mathrm{MCT}\}$ \\
\hline 2RFD & $\{2 \mathrm{CA}, \mathrm{RC}, \mathrm{DC}, \mathrm{MCR}, \mathrm{SCT}\}$ \\
\hline 2RFDFS & $\{2 \mathrm{CA}, \mathrm{RC}, \mathrm{DC}, \mathrm{MCR}, \mathrm{MCT}\}$ \\
\hline FR1D & $\{\mathrm{NCA}, \mathrm{RC}, \mathrm{NDC}, \mathrm{MCR}, \mathrm{SCT}\}$ \\
\hline FRFD & $\{\mathrm{NCA}, \mathrm{RC}, \mathrm{DC}, \mathrm{MCR}, \mathrm{SCT}\}$ \\
\hline
\end{tabular}

Fig. 2. Minimum Cost Constraint Sets

Fig. 3 shows the transitions between the different system connectivity models for various constraint changes. Transitions are in the direction of decreased system resource constraints. Transitions that do not improve the system connectivity or that do not follow the minimum cost constraint sets are not shown.

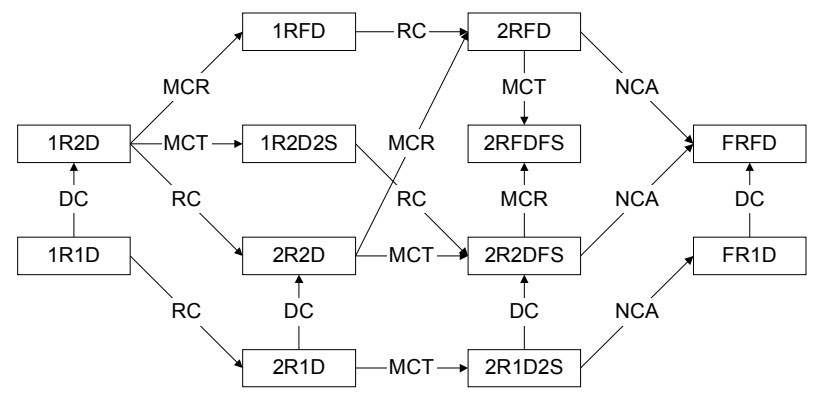

Fig. 3. Transition between System Connectivity Models

Figs. 4-15 show examples for each model with the chosen channel allocation in brackets. The given channel allocations are chosen to highlight the connectivity differences between models. Other channel allocations are possible, in some cases with better performance. The following discussion indicates where an alternate channel allocation would correspond to a distributed spatial diversity technique from the literature.

Single Relay Single Destination (1R1D): Each relay is connected to one transmitter and the destination is connected to one transmitter.

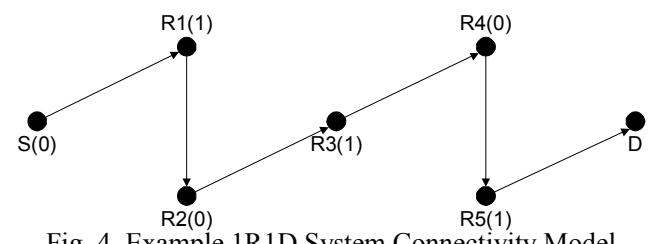

Fig. 4. Example 1R1D System Connectivity Model

The 1R1D model is minimally connected, and corresponds to the multihop models presented in $[3,6,15]$.

Single Relay 2Chnl Destination (1R2D): Each relay is connected to one transmitter and the destination is connected to a subset of transmitters on one channel.

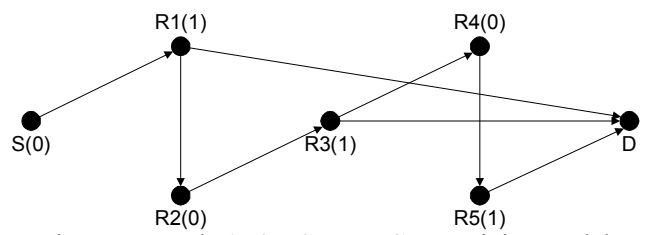

Fig. 5. Example 1R2D System Connectivity Model
The 1R2D model corresponds to the multi-user diversity models presented in $[1,5]$ under an alternate channel allocation where the source transmits on channel $\mathrm{C} 0$ and all relays transmit on channel $\mathrm{C} 1$. The source and destination are connected to all relays.

Single Relay 2Chnl Destination 2Chnl Source (1R2D2S): Each relay is connected to one transmitter, the destination is connected to a subset of transmitters on one channel, and the source is connected to a subset of receivers on both channels.

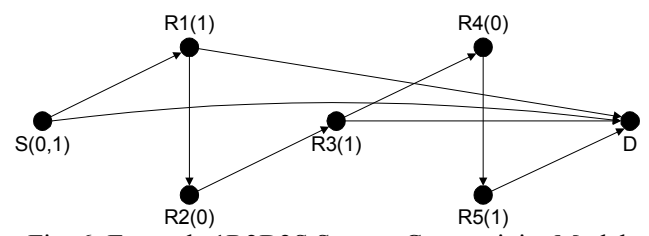

Fig. 6. Example 1R2D2S System Connectivity Model

The 1R2D2S model has identical connectivity to the cooperative diversity models presented in [8-11,13-14] under an alternate channel allocation where the source transmits on $\mathrm{C} 0$ and $\mathrm{C} 1$ and all relays transmit on $\mathrm{C} 1$. The source and destination are connected to all relays and to each other.

Single Relay Full Destination (1RFD): Each relay is connected to one transmitter and the destination is connected to all transmitters.

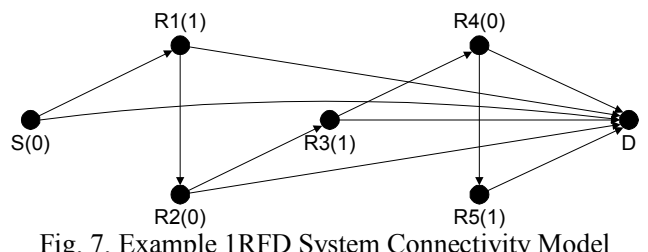

Fig. 7. Example 1RFD System Connectivity Model

The 1RFD model corresponds to the cooperative diversity models presented in [8-11,13-14] under an alternate channel allocation where the source transmits on $\mathrm{C} 0$ and all relays transmit on $\mathrm{C} 1$. The source and destination are connected to all relays and to each other.

2Chnl Relay Single Destination (2R1D): Each relay is connected to a subset of transmitters on one channel and the destination is connected to one transmitter.

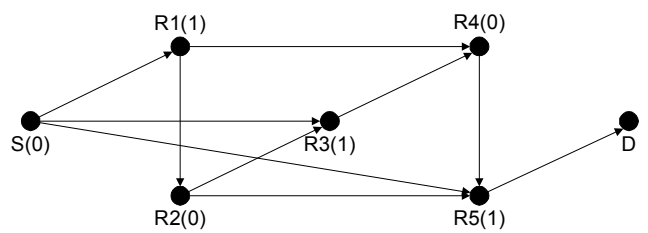

Fig. 8. Example 2R1D System Connectivity Model

2Chnl Relay Single Destination 2Chnl Source (2R1D2S): Each relay is connected to a subset of transmitters on one channel, the destination is connected to one transmitter, and the source is connected to a subset of receivers on both channels.

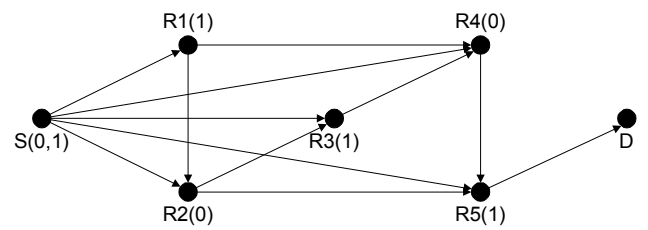

Fig. 9. Example 2R1D2S System Connectivity Model 
2Chnl Relay 2Chnl Destination (2R2D): Each relay is connected to a subset of transmitters on one channel and the destination is connected to a subset of transmitters on one channel.

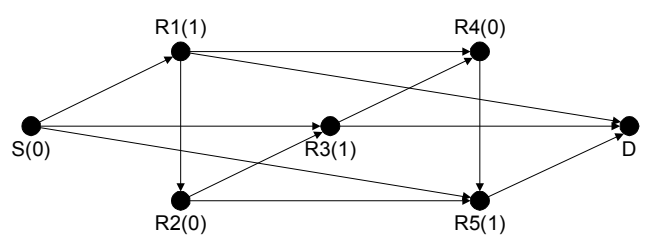

Fig. 10. Example 2R2D System Connectivity Model

2Chnl Relay 2Chnl Destination Full Source (2R2DFS): Each relay is connected to a subset of transmitters on one channel, the destination is connected to a subset of transmitters on one channel, and the source is connected to all receivers.

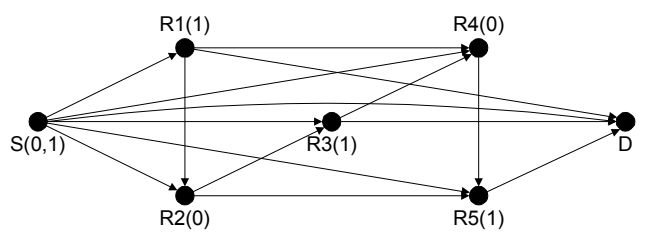

Fig. 11. Example 2R2DFS System Connectivity Model

2Chnl Relay Full Destination (2RFD): Each relay is connected to a subset of transmitters on one channel and the destination is connected to all transmitters.

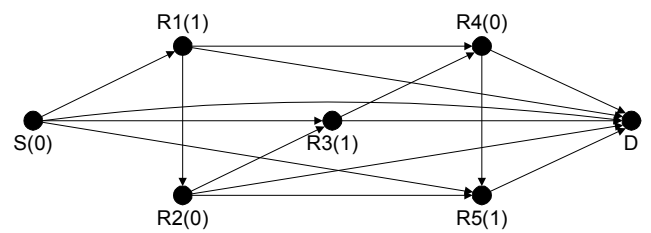

Fig. 12. Example 2RFD Connectivity Model

The 2RFD model effectively extends the cooperative diversity models presented in $[8-11,13-14]$ to the case where the relays belong to different cooperation groups in multiple tiers between the source and destination.

2Chnl Relay Full Destination Full Source (2RFDFS): Each relay is connected to a subset of transmitters on one channel, the destination is connected to all transmitters, and the source is connected to all receivers.

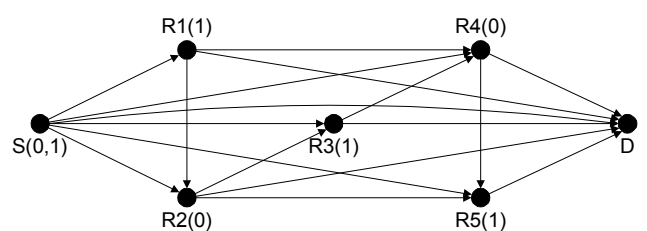

Fig. 13. Example 2RFDFS System Connectivity Model

Full Relay Single Destination (FRID): Each relay is connected to all transmitters and the destination is connected to one transmitter.

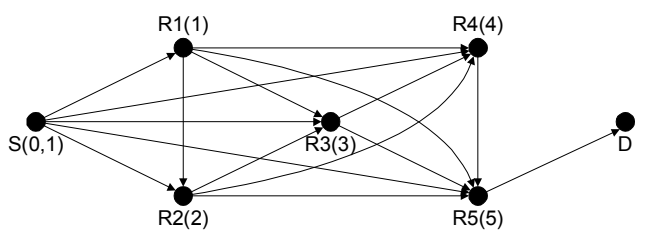

Fig. 14. Example FR1D System Connectivity Model
Full Relay Full Destination (FRFD): Each relay is connected to all transmitters and the destination is connected to all transmitters.

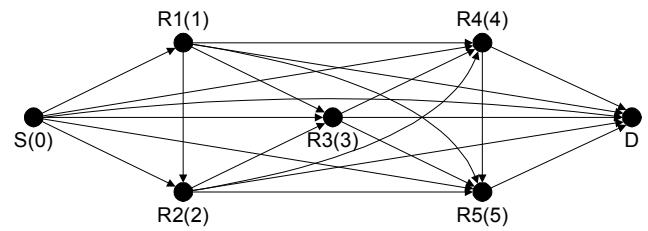

Fig. 15. Example FRFD System Connectivity Model

The FRFD model is fully connected, and corresponds to the multihop diversity models presented in $[2,3]$.

\section{Simulation Results}

The system connectivity models are applied in a series of simulations that allow the performance impact of the individual system resource constraints to be isolated. The singlehop channel is shown for reference. A BPSK modulation scheme is used for simplicity of exposition. The simulations use equations (6), (10), (13), and (15) from [3] and (9) from [4] with a propagation exponent of 4, equal power for all transmitting terminals, and total transmit power that is constrained to the transmit power of the singlehop reference channel. Maximal ratio combining is assumed.

Figs. 17-19 respectively compare the BER of the system connectivity models for three relaying methods. For amplified relaying each relay terminal combines and amplifies the received signals before retransmission. For decoded relaying with error propagation each relay terminal combines, digitally decodes, and re-encodes the received signals before retransmission and decoding errors at relays propagate as decoding errors at the destination. For decoded relaying without error propagation each relay terminal combines, digitally decodes, and re-encodes the received signals before retransmission and decoding errors at relays do not propagate as decoding errors at the destination. This is effectively an upper bound on the performance of decoded relaying and corresponds to an error-free version of the adaptive decode-and-forward protocol presented in [7,9-11].

The terminal distribution is that shown in Figs. 4-15 with network connectivity that optimizes the error performance for each system connectivity model and relaying method. The topology is symmetric with normalized link distances:

$$
d_{S, D}=3 d_{S, R 1}=2 d_{S, R 3}=\frac{5}{4} d_{S, R 4}=3 d_{R 1, R 2}=2 d_{R 1, R 4}=\frac{3}{2} d_{R 1, R 5} .
$$

Fig. 16 summarizes the impact of the constraints on the performance of the system for the three relaying methods.

\begin{tabular}{|l|l|l|l|l|l|}
\hline Relaying Method & \multicolumn{1}{|c|}{ NCA } & \multicolumn{1}{|c|}{ RC } & \multicolumn{1}{c|}{ DC } & \multicolumn{1}{|c|}{ MCR } & \multicolumn{1}{|c|}{ MCT } \\
\hline Amplified & Small & Small & Large & Large & Small \\
\hline Decoded w Prop & Small & Medium & Medium & Small & Medium \\
\hline Decoded w/o Prop & Small & Medium & Large & Large & Small \\
\hline
\end{tabular}

Fig. 16. Impact of System Resource Constraints

\section{CONCLUSION}

This paper considers the impact of various system resource constraints on the connectivity and performance of relaying channels. System connectivity models are derived from the constraint combinations, and minimum cost constraint sets are given. The relationship of the models to the distributed 
spatial diversity techniques presented in the literature [1-5,811,13-14] are noted. Simulations are presented that show the impact of the individual system resource constraints on amplified relaying, decoded relaying with error propagation, and decoded relaying without error propagation.

The impact of the constraints is different for each relaying method. The results indicate that for amplified relaying and decoded relaying without error propagation the priority is to maximize the connectivity of the destination terminal, while for decoded relaying with error propagation the priority is to equalize the connectivity of the destination and relay terminals. For amplified relaying the diversity order of the system is dependent on the connectivity of the destination. For decoded relaying with error propagation the diversity order of the system is constrained by the connectivity of the minimally connected relay and therefore limited to one. For decoded relaying without error propagation the diversity order of the system is dependent on the connectivity of both destination and relays.

The results provide general guidance for the order in which the constraints should be lifted to maximize connectivity and performance. For amplified relaying the constraints should be lifted in the following order: DC, MCR, IC, RC, and MCT. For decoded relaying with error propagation the constraints should be lifted in the following order: IC, RC, DC, MCT, and MCR. For decoded relaying without error propagation the constraints should be lifted in the following order: DC, MCR, IC, RC, and MCT. Since the impact of lifting the NCA constraint when all other constraints are lifted is small, but increases the system cost significantly, it is not expected that having a separate channel available for each terminal will be implemented in practice. Additional simulations have shown that this guidance holds for other network topologies.

\section{REFERENCES}

[1] P. Anghel, G. Leus, and M. Kaveh, "Multi-User Space-Time Coding in Cooperative Networks," Proc. of IEEE International Conference on Acoustics, Speech, and Signal Processing, 2003.

[2] J. Boyer, D. D. Falconer, and H. Yanikomeroglu, "A Theoretical Characterization of Multihop Wireless Communications Channels With Diversity," Proc. of IEEE GLOBECOM, 2001.

[3] J. Boyer, D. D. Falconer, and H. Yanikomeroglu, "Multihop Diversity in Wireless Relaying Channels," IEEE Trans. on Communications, vol. 52, no. 10, 2004.

[4] J. Boyer, D. D. Falconer, and H. Yanikomeroglu, "On the Aggregate SNR of Amplified Relaying Channels," Proc. of IEEE GLOBECOM, 2004.

[5] V. Emamian, P. Anghel, and M. Kaveh, "Multi-User Spatial Diversity in an Shadow-Fading Environment," Proc. of IEEE Vehicular Technology Conference, 2002.

[6] M. Hasna and M. Alouini, "End-to-End Performance of Transmission Systems with Relays over Rayleigh Fading Channels," IEEE Trans. on Wireless Communications, vol. 2, no. 6, 2003.

[7] P. Herhold, E. Zimmermann, G. Fettweis, "A Simple Cooperative Extension to Wireless Relaying," Proc. of Int. Zurich Seminar on Communications, 2004.

[8] J. Laneman and G. Wornell, "Energy-Efficient Antenna Sharing and Relaying for Wireless Networks," Proc. of IEEE Wireless Communications and Networking Conference, 2000.

[9] J. Laneman and G. Wornell, "Exploiting Distributed Spatial Diversity in Wireless Networks," Proc. of Allerton Conference on Communications, Control, Computing, 2000.

[10] J. Laneman G. Wornell, "Distributed Space-Time-Code Protocols for Exploiting Cooperative Diversity in Wireless Networks," IEEE Trans. on Information Theory, vol. 49, no. 10, 2003.
[11] J. Laneman, D. Tse, and G. Wornell, "Cooperative Diversity in Wireless Networks: Efficient Protocols and Outage Behavior," IEEE Trans. on Information Theory, vol. 50, no. 12, 2004.

[12] A. Ribeiro, X. Cai, and G. Giannakis, "Symbol Error Probabilities for General Cooperative Links," Proc. of IEEE International Conference on Communications, 2004.

[13] A. Sendonaris, E. Erkip, and B. Aazhang, "User Cooperation Diversity - Part I: System Description," IEEE Trans. on Communications, vol. 51 , no. $11,2003$.

[14] A. Sendonaris, E. Erkip, and B. Aazhang, "User Cooperation Diversity - Part II: Implementation Aspects and Performance Analysis," IEEE Trans. on Communications, vol. 51, no. 11, 2003.

[15] L. Yang, H. Hasna, and M. Alouini, "Average Outage Duration of Multihop Communication Systems with Regenerative Relays," Proc. of IEEE Vehicular Technology Conference, 2003.

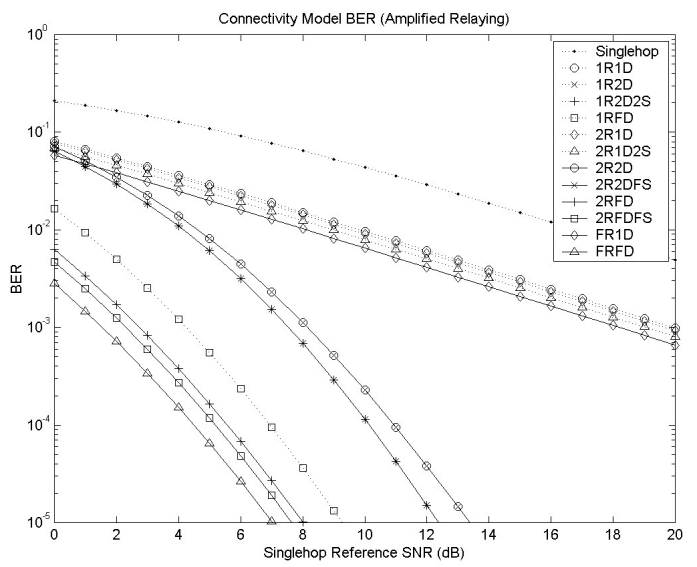

Fig. 17. Amplified Relaying Impact Comparison

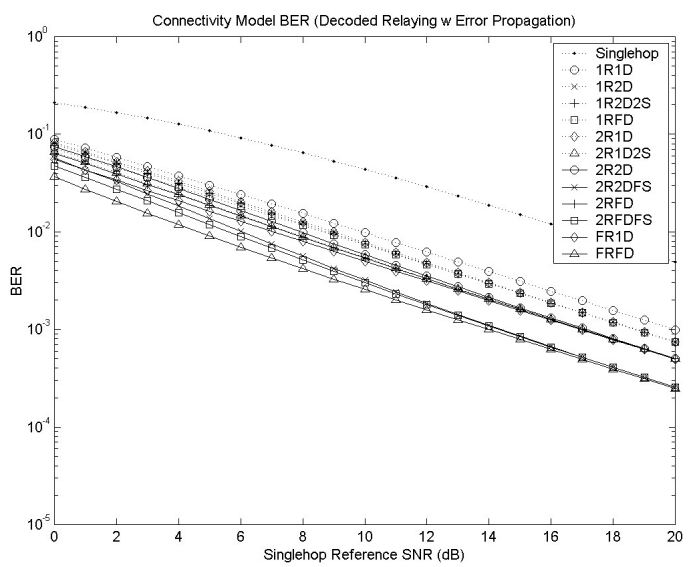

Fig. 18. Decoded Relaying w Propagation Impact Comparison

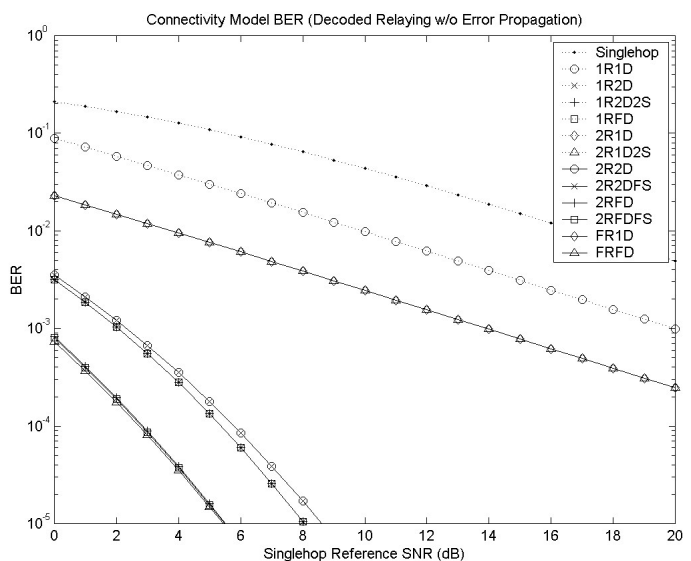

Fig. 19. Decoded Relaying w/o Propagation Impact Comparison 